\title{
Vitamin D status and seroconversion for COVID-19 in UK healthcare workers
}

To the Editor:

The coronavirus disease 2019 (COVID-19) pandemic is a global health emergency, resulting in over 50 million infections and over 1.2 million deaths as of mid-November 2020 [1]. Healthcare workers are at a high risk of COVID-19 with large numbers of deaths reported around Europe and the UK, particularly among staff in the Black, Asian and minority ethnic (BAME) demographic group [2]. COVID-19 has disproportionately affected BAME individuals even after accounting for age, sex, social deprivation and comorbidity [3].

Vitamin D deficiency (VDD) is common in those of BAME ethnicity [4]. In VDD, both innate and adaptive immunity becomes dysregulated, increasing the risk of respiratory infection, as seen in influenza, common cold viruses and tuberculosis $[5,6]$. There has been a great deal of interest in the role of VDD in COVID-19, with recent evidence suggesting VDD is more frequent in patients with severe COVID-19 compared to mild cases [7]. The prevalence of VDD and association with COVID-19 in healthcare workers has not been investigated.

We hypothesised that VDD was more common in healthcare workers who have seroconverted for COVID-19. This study defined the factors associated with VDD and the relationship between COVID-19 seroconversion and VDD.

This cross-sectional observational study recruited healthcare workers between 12 and 22 May, 2020 from the University Hospitals Birmingham NHS Foundation Trust (UHBFT) across four sites. This was a sub-study of the COVID-19 convalescent immunity study (COCO) approved by the London - Camden and Kings Cross Research Ethics Committee (20/HRA/1817). The main inclusion criteria were staff members who had isolated for symptoms suggestive of COVID-19. After obtaining consent, blood samples were taken for measurement of vitamin D levels by mass spectrometry, and anti-SARS-CoV-2 spike glycoprotein antibodies using a combined IgG, IgA, IgM ELISA (The Binding Site; product code: MK654) [8]. This CE marked assay has $98.6 \%$ (95\% CI 92.6-100) sensitivity and 98.3\% (95\% CI 96.4-99.4) specificity. The median time from symptom onset to sample collection was 48 days. COVID-19 seroconversion was used to indicate previous infection with severe acute respiratory syndrome coronavirus 2 (SARS-CoV-2). VDD was defined as serum $25(\mathrm{OH}) \mathrm{D}_{3}$ concentration $<30 \mathrm{nmol} \cdot \mathrm{L}^{-1}$ as per the UHBFT clinical laboratory reference range and the UK National Osteoporosis Society guidance [9].

Of the 392 healthcare workers studied, 61 (15.6\%) had VDD. The participant demographics, occupation and seroconversion status are displayed in table 1 . Those with VDD were significantly more likely to be BAME $(p<0.0001)$ and in a junior doctor job role $(p=0.029)$; there were no differences in age, body mass index or comorbidity status between those with VDD and those without. Using backwards logistic regression to determine factors associated with $\mathrm{VDD}$, the multivariate analysis used all demographic variables in table 1 . The significant independent factors for VDD were BAME (OR 8.86, 95\% CI 4.7516.52; $\mathrm{p}<0.001$ ) and seroconversion (OR 2.15, 95\% CI 1.11-4.17; $\mathrm{p}=0.023)$. The overall predictive power of the model was $77.9 \%$ (95\% CI 71.1-84.7, SE 3.5\%; $\mathrm{p}<0.001)$ indicated by the area under the receiver operator characteristic (ROC) curve.

Self-reported symptoms in healthcare workers $(n=386)$, included fever $(n=235,61 \%)$, breathlessness $(\mathrm{n}=186,48 \%)$, cough $(\mathrm{n}=117,30 \%)$, loss of smell/taste $(\mathrm{n}=169,44 \%)$, body aches/pains $(\mathrm{n}=274,71 \%)$, fatigue $(n=339,88 \%)$, diarrhoea $(n=115,30 \%)$ and sore throat $(n=197,51 \%)$. VDD staff experienced more 
TABLE 1 Participant demographic, occupation and seroconversion status

\begin{tabular}{|c|c|c|c|c|}
\hline & Total & $\begin{array}{l}\text { Vitamin D } \\
\text { deficient }\end{array}$ & $\begin{array}{c}\text { Non-vitamin D } \\
\text { deficient }\end{array}$ & $\mathrm{p}$-value \\
\hline Subjects $n$ & 392 & 61 & 331 & \\
\hline Age years & $41(30-50)$ & $35(28-47.5)$ & $42(31-50)$ & 0.073 \\
\hline \multicolumn{5}{|l|}{ Gender } \\
\hline Female & $285(73 \%)$ & $40(66 \%)$ & 245 (74\%) & \multirow[t]{3}{*}{0.112} \\
\hline Male & $100(26 \%)$ & $21(34 \%)$ & $79(24 \%)$ & \\
\hline Not stated & $7(2 \%)$ & & $7(2 \%)$ & \\
\hline BMI $\mathrm{kg} \cdot \mathrm{m}^{-2}$ & $25.9(22.9-30.1)$ & $25.4(22.9-30.8)$ & $26.0(22.9-30.1)$ & 0.794 \\
\hline \multicolumn{5}{|l|}{ Ethnicity } \\
\hline White & $279(71 \%)$ & $18(30 \%)$ & $261(79 \%)$ & \multirow[t]{3}{*}{$<0.0001$} \\
\hline BAME & $108(28 \%)$ & $43(70 \%)$ & $65(20 \%)$ & \\
\hline Not stated & $5(1 \%)$ & & $5(2 \%)$ & \\
\hline \multicolumn{5}{|l|}{ Comorbidities } \\
\hline None & $240(61 \%)$ & $40(66 \%)$ & $200(60 \%)$ & \multirow[t]{2}{*}{0.478} \\
\hline One or more & 152 (39\%) & 21 (34\%) & 131 (40\%) & \\
\hline \multicolumn{5}{|l|}{ Job role } \\
\hline Junior doctor & $50(13 \%)$ & $15(25 \%)$ & $35(11 \%)$ & \multirow[t]{9}{*}{$0.029^{\#}$} \\
\hline Consultant & $65(17 \%)$ & $7(11 \%)$ & $58(18 \%)$ & \\
\hline Junior nurse & $65(17 \%)$ & $12(20 \%)$ & $53(16 \%)$ & \\
\hline Senior nurse & $66(17 \%)$ & $7(11 \%)$ & $59(18 \%)$ & \\
\hline Physiotherapist & $28(7 \%)$ & $4(7 \%)$ & $24(7 \%)$ & \\
\hline Laboratory worker & $26(7 \%)$ & $7(11 \%)$ & $19(6 \%)$ & \\
\hline $\begin{array}{l}\text { Radiology/theatre staff/ } \\
\text { pharmacy }\end{array}$ & $21(5 \%)$ & $1(2 \%)$ & $20(6 \%)$ & \\
\hline Secretary/administrator & $35(9 \%)$ & $2(3 \%)$ & $33(10 \%)$ & \\
\hline $\begin{array}{l}\text { Healthcare assistant/ } \\
\text { phlebotomist }\end{array}$ & $36(9 \%)$ & $6(10 \%)$ & $30(9 \%)$ & \\
\hline \multicolumn{5}{|l|}{ Seroconversion } \\
\hline Yes & $214(55 \%)$ & $44(72 \%)$ & $170(51 \%)$ & \multirow[t]{2}{*}{0.003} \\
\hline No & $178(45 \%)$ & $17(28 \%)$ & $161(49 \%)$ & \\
\hline
\end{tabular}

Data are presented as median (interquartile range) or $\mathrm{n}(\%)$, unless otherwise indicated. Vitamin $\mathrm{D}$ deficient is Serum $25(\mathrm{OH}) \mathrm{D}_{3}<30 \mathrm{nmol} \cdot \mathrm{L}^{-1}$, while not deficient is $\geqslant 30 \mathrm{nmol} \cdot \mathrm{L}^{-1}$. Where proportions are shown, they were calculated using the $\mathrm{n}$ numbers shown in columns as denominator; $\mathrm{p}$-values were calculated using Mann-Whitney test for data presented as median and interquartile range, and by Fisher's exact test for data presented as $\mathrm{n}(\%)$, with $p<0.05$ considered significant. The sample size for the backward logistic regression is 379 , with 13 excluded from this analysis; seven because gender was not stated, five due to ethnicity not stated and one with body mass index (BMI) unknown. Age and BMI were treated as continuous variables, job role as nine categories, while all others were treated as dichotomous. Eight participants were taking vitamin D supplements, one in the vitamin D deficient group and seven in the non-vitamin $\mathrm{D}$ deficient group. Comorbidities were classified as shown because they were uncommon in this cohort, with the most common comorbidities being hypertension $(n=34,9 \%)$ and asthma $(n=26,7 \%)$. \#: p-value of 0.226 when excluding junior doctor group in analysis. BAME: Black, Asian and minority ethnic demographic group.

body aches/pains ( $82 \%$ versus $69 \%$; $=0.045)$ but there were no significant differences in other symptoms reported between groups.

Seroconversion was higher in healthcare workers with VDD compared to those without ( $\mathrm{n}=44 / 61,72 \%$ versus $\mathrm{n}=170 / 331,51 \%$; $\mathrm{p}=0.003$ ), representing an absolute increase of 13 cases in the VDD group. Seroconversion was higher in BAME males with VDD compared to those without ( $\mathrm{n}=17 / 18,94 \%$ versus $\mathrm{n}=12 / 23$, 52\%; $\mathrm{p}=0.005$ ); no differences were observed in the other ethnic-gender sub-analysis. Using backwards logistic regression to determine factors associated with seroconversion, the multivariate analysis used all the variables from table 1; only VDD was a significant independent risk factor for developing seroconversion (OR 2.6, 95\% CI 1.41-4.80; $\mathrm{p}=0.002$ ). The overall predictive power of the model was $55.5 \%$ (95\% CI 49.8-61.2, SE 2.9\%; $\mathrm{p}=0.06$ ) as indicated by the area under the ROC curve.

To our knowledge this is the first study to investigate VDD prevalence in a UK healthcare worker cohort. VDD was relatively uncommon (15.6\%) and lower than healthcare worker studies published in the USA and Gulf Areas, which may in part reflect differences in reference ranges and vitamin D assays used [10]. 
The increase in VDD seen in junior doctors echoes previous findings of junior doctors have lower levels than senior doctors [10], which may be due to increased frontline work and different shift patterns.

Our data supports previous findings of higher VDD in BAME ethnicity [4]. While BAME was not an independent risk factor for seroconversion, our sub-group analysis found that VDD BAME males may be most at risk from COVID-19 as there was remarkably high seroconversion rate of $94 \%$ in this sub-group. Although this is a cohort of mild COVID-19, being BAME and male are known risk factors for a severe outcome from COVID-19.

Our study showing VDD as an independent risk factor for COVID-19 seroconversion is consistent with others including a large US study which found that COVID-19 positivity was inversely related to patient vitamin $\mathrm{D}$ levels in the preceding 12 months [11]. Additional data from Israel found low vitamin D increased the risk of COVID-19 positivity and COVID-19 related hospitalisation [12]. Furthermore, a recent open labelled clinical trial from Spain provided a proof of concept that high-dose vitamin D treatment may be useful therapy for severe COVID-19 [13].

The role of vitamin D in modulating the immune response to COVID-19 is likely to be multifactorial. Vitamin D increases the production of antimicrobial peptides in the respiratory epithelium, which may protect against viral infection. Also, vitamin $\mathrm{D}$ supplementation has been shown to reduce viral upper respiratory tract infections in metanalysis [14]. Vitamin D may help reduce the body's response to COVID-19; older patients with VDD $\left(<30 \mathrm{nmol} \cdot \mathrm{L}^{-1}\right)$ had higher D-dimer levels, a marker of inflammation and vascular damage, and required more noninvasive ventilation support [15]. There is a bidirectional association between VDD and COVID-19 in our study and therefore it is unclear if VDD is a cause and/ or consequence of COVID-19.

This study has several limitations. Firstly, staff were recruited from a single NHS trust, although it is the second largest in the UK, spanning four hospital sites. Secondly, as the healthcare workers had mild COVID-19, this study does not inform of the role of VDD in severe COVID-19. Thirdly, due to relatively small sample size, we were unable to analyse the differences between staff from different BAME sub-groups. Fourthly, there is a risk of bias from recruiting individuals with self-reported symptoms compared to a cross-sectional survey of all healthcare workers. Finally, other confounders such as sociocultural factors which may affect risk of transmission and/or VDD were not addressed.

In summary, in healthcare workers who have isolated due to symptoms of COVID-19, those of BAME ethnicity are at the highest risk of VDD. Furthermore, VDD was an independent risk factor for development of COVID-19 seroconversion, with the biggest differences seen in the BAME male group. Therefore, we suggest future high-dose vitamin D treatment trials should target such at risk groups within healthcare workers with aim of potentially preventing or alleviating COVID-19.

Aduragbemi A. Faniyi ${ }^{1,5}$, Sebastian T. Lugg $^{1,5}$, Sian E. Faustini ${ }^{2}$, Craig Webster ${ }^{3}$, Joanne E. Duffy ${ }^{3}$, Martin Hewison $^{4}$, Adrian Shields $\circledast^{2,3}$, Peter Nightingale ${ }^{3}$, Alex G. Richter ${ }^{2,3,6}$ and David R. Thickett ${ }^{1,3,6}$

${ }^{1}$ Birmingham Acute Care Research Group, Institute of Inflammation and Ageing, University of Birmingham, Birmingham, UK. ${ }^{2}$ Clinical Immunology Service, Institute of Immunology and Immunotherapy, University of Birmingham, Birmingham, UK. ${ }^{3}$ University Hospitals Birmingham NHS Foundation Trust, Birmingham, UK. ${ }^{4}$ Institute of Metabolism and Systems Research, University of Birmingham, Birmingham, UK. ${ }^{5}$ Joint first authors. ${ }^{6}$ Joint last authors.

Correspondence: David R. Thickett, Institute of Inflammation and Ageing, University of Birmingham, Birmingham B15 2TH, UK. E-mail: d.thickett@bham.ac.uk

Received: 15 Oct 2020 | Accepted: 18 Nov 2020

Acknowledgements: We thank the staff of University Hospitals Birmingham NHS Foundation Trust who kindly volunteered for this study. We would also like to thank the research staff of the Birmingham Wellcome NIHR Clinical Research Facility who undertook the staff facing assessments. We would like to thank colleagues at the Clinical Immunology Service for overseeing recruitment and sample processing. We also thank our colleagues Prof Adrian Martineau from Queen Mary University of London, Prof Elizabeth Sapey, Dr Dhruv Parekh, both from University of Birmingham and Prof Jon Rhodes from University of Liverpool, who have given helpful feedback on the study results and manuscript.

Author contributions: A.G. Richter and D.R. Thickett conceptualised the study. A.A. Faniyi, S.E. Faustini, C. Webster, J.E. Duffy, A. Shields, A.G. Richter and D.R. Thickett contributed to data acquisition. A.A. Faniyi, S.T. Lugg, P. Nightingale and D.R. Thickett analysed the data. All authors contributed to data interpretation. A.A. Faniyi, S.T. Lugg and D.R. Thickett drafted the manuscript. All authors contributed to the review and approval of the final copy of the manuscript.

Conflict of interest: A.A. Faniyi has nothing to disclose. S.T. Lugg has nothing to disclose. S.E. Faustini has nothing to disclose. C. Webster has nothing to disclose. J.E. Duffy has nothing to disclose. M. Hewison reports personal fees for 
lectures from Thornton Ross, outside the submitted work. A. Shields has nothing to disclose. P. Nightingale has nothing to disclose. A.G. Richter has nothing to disclose. D.R. Thickett reports personal fees for lectures from Thornton Ross, outside the submitted work.

Support statement: This study was funded internally by the University of Birmingham and University Hospitals Birmingham NHS Foundation Trust and supported by the National Institute for Health Research (NIHR)/Wellcome Trust Birmingham Clinical Research Facility. A.A. Faniyi and D.R. Thickett are funded by the Medical Research Council (MR/S002782/1). The Binding Site (Edgbaston, UK) have provided reagents and plates for the SARS-CoV-2 ELISA free of charge. Funding information for this article has been deposited with the Crossref Funder Registry.

\section{References}

1 World Health Organization. Coronavirus Disease (COVID-19) Dashboard. https://covid19.who.int Date last updated: 13 Nov 2020. Date last accessed: 13 Nov 2020.

2 Health Service Journal. Exclusive: Deaths of NHS Staff from Covid-19 Analysed. www.hsj.co.uk/exclusive-deathsof-nhs-staff-from-covid-19-analysed/7027471.article Date last updated: 22 Apr 2020. Date last accessed: 13 Nov 2020.

3 Office for National Statistics. Coronavirus (COVID-19) Related Deaths by Ethnic Group, England and Wales: 2 March 2020 to 10 April 2020. www.ons.gov.uk/peoplepopulationandcommunity/birthsdeathsandmarriages/deaths/ articles/coronavirusrelateddeathsbyethnicgroupenglandandwales/2march2020to10april2020 Date last updated: 7 May 2020. Date last accessed: 13 Nov 2020.

4 Webster C. Relationship of total 25-OH vitamin D concentrations to indices of multiple deprivation: geoanalysis of laboratory results. Ann Clin Biochem 2013; 50: 31-38.

5 Greiller CL, Martineau AR. Modulation of the immune response to respiratory viruses by vitamin D. Nutrients 2015; 7: 4240-4270.

6 Nnoaham KE, Clarke A. Low serum vitamin D levels and tuberculosis: a systematic review and meta-analysis. Int Epidemiol 2008; 37: 113-119.

7 Panagiotou G, Tee SA, Ihsan Y, et al. Low serum 25-hydroxyvitamin D (25[OH]D) levels in patients hospitalized with COVID-19 are associated with greater disease severity. Clin Endocrinol (Oxf) 2020; 93: 508-511.

8 Faustini SE, Jossi SE, Perez-Toledo M, et al. Detection of antibodies to the SARS-CoV-2 spike glycoprotein in both serum and saliva enhances detection of infection. medRxiv 2020; preprint [https://doi.org/10.1101/2020.06.16. 20133025].

9 Aspray TJ, Bowring C, Fraser W, et al. National Osteoporosis Society vitamin D guideline summary. Age Ageing 2014; 43: 592-595.

10 Sowah D, Fan X, Dennett L, et al. Vitamin D levels and deficiency with different occupations: a systematic review. BMC Public Health 2017; 17: 519.

11 Kaufman HW, Niles JK, Kroll MH, et al. SARS-CoV-2 positivity rates associated with circulating 25-hydroxyvitamin D levels. PLoS One 2020; 15: e0239252.

12 Merzon E, Tworowski D, Gorohovski A, et al. Low plasma 25(OH) vitamin D level is associated with increased risk of COVID-19 infection: an Israeli population-based study. FEBS J 2020; 287: 3693-3702.

13 Entrenas Castillo M, Entrenas Costa LM, Vaquero Barrios JM, et al. Effect of calcifediol treatment and best available therapy versus best available therapy on intensive care unit admission and mortality among patients hospitalized for COVID-19: a pilot randomized clinical study. J Steroid Biochem Mol Biol 2020; $203: 105751$.

14 Martineau AR, Jolliffe DA, Hooper RL, et al. Vitamin D supplementation to prevent acute respiratory tract infections: systematic review and meta-analysis of individual participant data. BMJ 2017; 356: i6583.

15 Baktash V, Hosack T, Patel N, et al. Vitamin D status and outcomes for hospitalised older patients with COVID-19. Postgrad Med J 2020; in press [https://doi.org/10.1136/postgradmedj-2020-138712]. 